\title{
Case fatality of COPD exacerbations: a meta-analysis and statistical modelling
} approach

\author{
M. Hoogendoorn*, R.T. Hoogenveen\#, M.P. Rutten-van Mölken*, J. Vestbo ${ }^{\uparrow,+, \S}$ and \\ T.L. Feenstra ${ }^{\#, f}$
}

ABSTRACT: The aim of our study was to estimate the case fatality of a severe exacerbation from long-term survival data presented in the literature.

A literature search identified studies reporting $\geqslant 1.5 \mathrm{yr}$ survival after a severe chronic obstructive pulmonary disease (COPD) exacerbation resulting in hospitalisation. The survival curve of each study was divided into a critical and a stable period. Mortality during the stable period was then estimated by extrapolating the survival curve during the stable period back to the time of exacerbation onset. Case fatality was defined as the excess mortality that results from an exacerbation and was calculated as 1 minus the (backwardly) extrapolated survival during the stable period at the time of exacerbation onset. The $95 \%$ confidence intervals $(\mathrm{CI})$ of the estimated case fatalities were obtained by bootstrapping. A random effect model was used to combine all estimates into a weighted average with $95 \% \mathrm{Cl}$.

The meta-analysis based on six studies that fulfilled the inclusion criteria resulted in a weighted average case-fatality rate of $15.6 \%(95 \% \mathrm{Cl} 10.9-20.3)$, ranging from $11.4 \%$ to $19.0 \%$ for the individual studies.

A severe COPD exacerbation requiring hospitalisation not only results in higher mortality risks during hospitalisation, but also in the time-period after discharge and contributes substantially to total COPD mortality.

KEYWORDS: Case fatality, chronic obstructive pulmonary disease, exacerbation, hospitalisation, meta-analysis

W orldwide, mortality due to chronic obstructive pulmonary disease (COPD) is high. According to the World Health Organization, at least 2.7 million deaths every year are due to COPD [1]. The 30-yr projections from the Global Burden of Disease Study show a striking increase in COPD as a cause of death to the third place worldwide in 2020 [2]. This increase largely results from a worldwide increase in the prevalence of smoking, especially in the developing countries and among females, and ageing of the population. The excess mortality among patients with COPD is high, not only because of the presence of COPD but also because of the increased prevalence of other smokingrelated diseases [3].

Many studies have analysed predictors of mortality in COPD. Among the factors independently associated with mortality in COPD are age, lung function (forced expiratory volume in $1 \mathrm{~s}$ and inspiratory capacity divided by total lung capacity), dyspnoea, comorbidity, body mass index (BMI), fat-free mass, exercise capacity, arterial oxygen tension, $\mathrm{C}$-reactive protein, the BODE index (BMI, the degree of airflow obstruction, dyspnoea and exercise capacity), airflow obstruction, dyspnoea and exercise capacity, and the number of previous hospitalisations [4, 5].

Because patients with COPD are often recorded as dying from other causes, it has been suggested that all-cause mortality is probably the best mortality measure to use in COPD [5]. Nevertheless, it is well known that many patients dying do so during a severe COPD exacerbation, when they experience acute respiratory failure [6]. However, there is a relative scarcity of knowledge on mortality rates from COPD exacerbations. Unlike in myocardial infarction and stroke [7], no estimates

\section{AFFILIATIONS}

*Institute for Medical Technology Assessment (IMTA), Erasmus University, Rotterdam,

${ }^{\#}$ National Institute for Public Health and the Environment (RIVM), Bilthoven,

${ }^{f}$ Dept of Epidemiology, University Medical Centre Groningen, Groningen, The Netherlands, "Respiratory Medicine Research Group, Manchester Academic Health Sciences Centre, Manchester, UK, +Dept of Cardiology and Respiratory Medicine, Hvidovre Hospital, and ${ }^{\text {s}}$ Faculty of Health Sciences, University of Copenhagen, Hvidovre Hospital, Hvidovre, Denmark.

\section{CORRESPONDENCE}

M. Hoogendoorn Institute for Medical Technology Assessment Erasmus University P.0. Box 1738 $3000 \mathrm{DR}$

Rotterdam

The Netherlands

E-mail: hoogendoorn@bmg.eur.nl

Received:

March 192010

Accepted after revision:

June 162010

First published online:

July 012010 


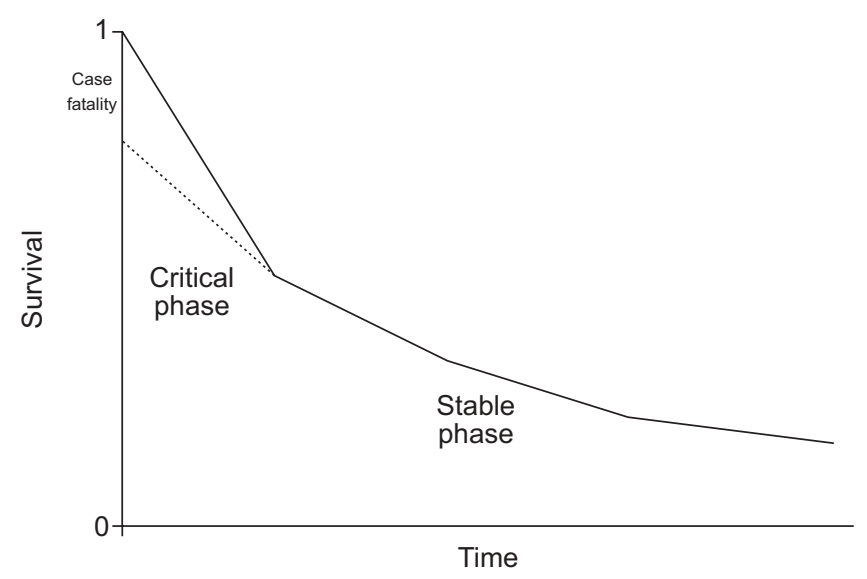

FIGURE 1. Survival curve after hospitalisation for an exacerbation of chronic obstructive pulmonary disease. ….... the extrapolated curve during the stable phase

of the case fatality of a COPD exacerbation exist. This may be associated with the absence of consensus on the length of the critical period during which the mortality risk is increased.

The most frequently reported outcome of death due to COPD exacerbations is short-term, in-hospital mortality [8]. Previous studies have estimated in-hospital mortality after hospitalisation for a COPD exacerbation to range from $2.5 \%$ to $14 \%$ [9, 10]. Mortality among patients admitted to intensive care units (ICUs) is much higher, i.e. up to $30 \%$ [11]. In-hospital mortality is insufficient to assess case fatality for at least two reasons. There is a selection bias towards patients with longer hospital stays and it does not incorporate the mortality that occurs after hospital discharge but is still attributable to the index exacerbation. Therefore, our study aimed to estimate the case fatality of a severe COPD exacerbation including the timeperiod after hospitalisation. This study arose out of our need to capture the impact of exacerbations on mortality within the context of a dynamic, COPD progression model $[12,13]$ used to evaluate the impact of different COPD interventions. To fully simulate the potential long-term impact of interventions which successfully prevent or treat exacerbations, the impact of severe exacerbations on mortality needed to be estimated. As the COPD population in the model is specified by age, which is a significant predictor of mortality in COPD [5], we also investigated the association between age and mortality after a severe exacerbation.

\section{METHODS AND MATERIALS}

We performed a comprehensive literature search in MEDLINE and EMBASE for journal articles published after 1990 reporting mortality or survival during and after hospitalisation for an exacerbation of COPD using the MESH (sub)headings "chronic obstructive pulmonary disease or COPD or chronic bronchitis" in combination with "mortality or dead or death* or life expectancy or survival or prognosis" and "hospital" or admission* or admitt* or exacerbation* or disease episodes". We also searched references listed from articles retrieved. Studies were excluded if the patient population was a

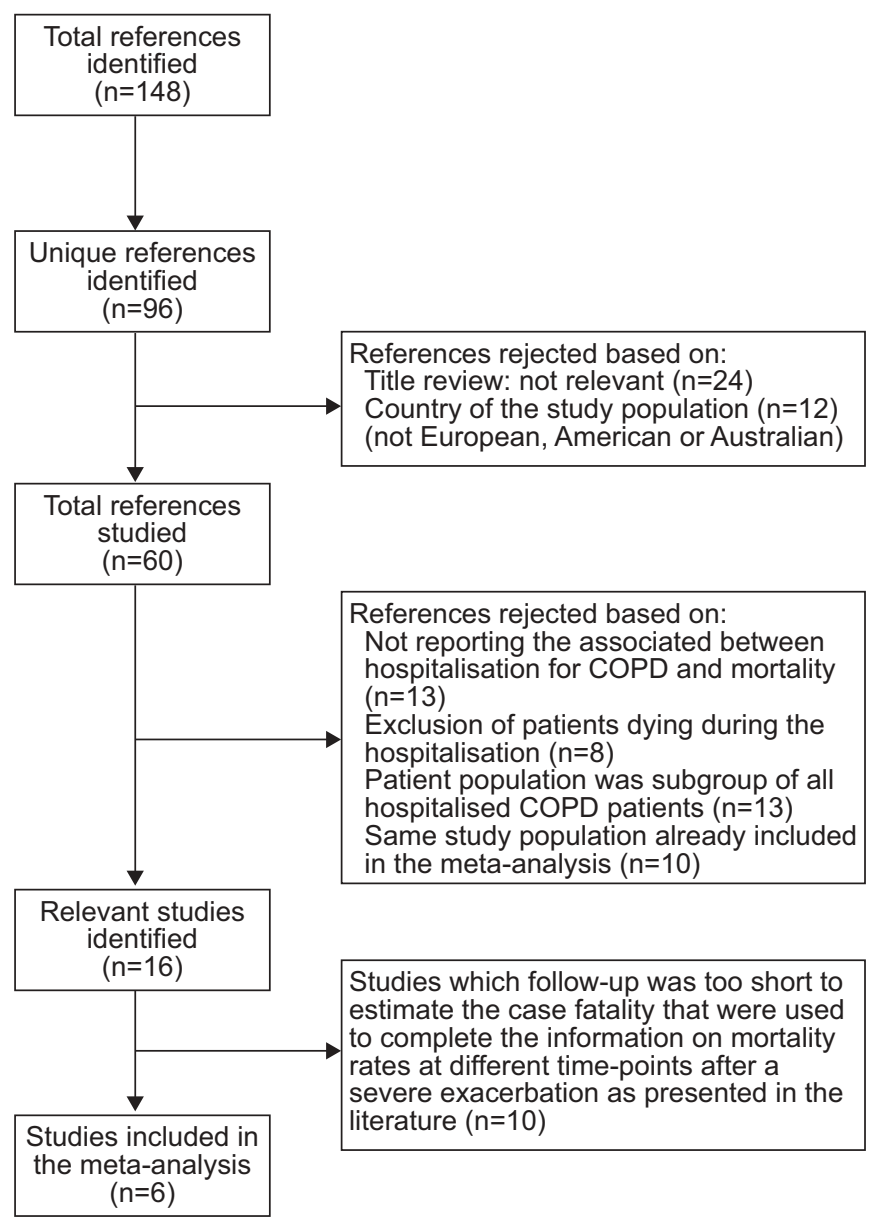

FIGURE 2. Results of the systemic literature search. COPD: chronic obstructive pulmonary disease.

subgroup of hospitalised COPD patients, such as patients requiring mechanical ventilation. Inclusion criteria were: a European, American or Australian study population; a followup period that started at hospital entry and lasted $\geqslant 1.5 \mathrm{yr}$; and presenting mortality rates at three or more time-points after hospital admission, or presenting a survival curve. Studies that fulfilled all inclusion criteria except for a follow-up of $1.5 \mathrm{yr}$ or the presence of three data points were used to complete the information on the average mortality rates at different timepoints after a severe exacerbation as presented in the literature. In addition to information on the average mortality rates at different time-points, data on the association between mortality and age was extracted from the studies.

Our general approach was as follows (fig. 1). For each study we extracted the survival curve presented in the article or estimated the curve from the presented data ourselves. We roughly distinguished between the critical and the stable period after hospital admission with the survival curve during the stable period being flatter than the one during the critical period. Several data points from the curve during the stable period were extracted to estimate survival during this period. Only data points well after the critical period were included. For each study the survival function during the stable period was then parameterised using three parameters. 


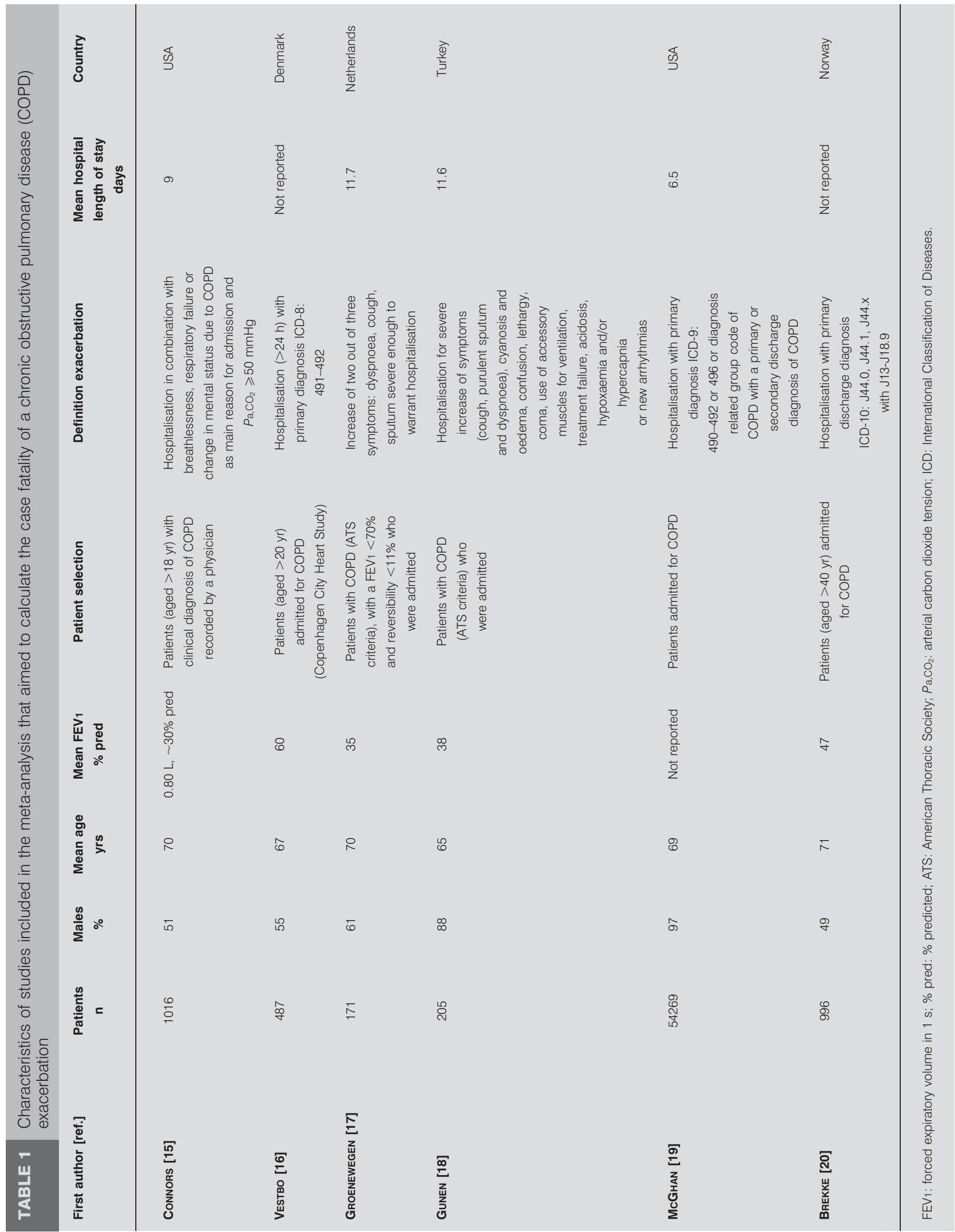




$$
S(t)=(1-g) \operatorname{Exp}\left[-\alpha t-\beta t^{2}\right]
$$

With $\mathrm{t}$ being the time, $\mathrm{t}=0$ being time of hospital admission, $S(t)$ the survival probability, $\alpha$ and $\beta$ the parameters that define the nonlinear change in survival over time, and $g$ the case fatality of the exacerbation.

The survival curve was fitted by minimising the sum of squared differences with the points that were extracted from the curve, or given in the publication. We then extrapolated the survival curve during the stable period back to the time of hospital admission and calculated where the curve intersected the vertical axis (i.e. the start of hospital admission). The case fatality was defined as the excess mortality that results from an exacerbation and equals: $g=1-S(0)$. Uncertainty intervals for each parameter were obtained from bootstrapping. Based on the given initial sample size and the calculated survival probabilities for each interval during the follow-up period, we randomly draw new survival numbers assuming binomial distributions. In this way we generated new survival curves, resulting in newly calculated values for the model parameters. The $2.5 \%$ and $97.5 \%$ percentile values correspond with the $95 \%$ uncertainty interval. Finally, estimates from all studies were combined to calculate the weighted average for $\mathrm{g}$, using random effect meta-analysis [14]. The weights were based on a combination of the sampling error (variance of case fatality within each study) and the random-effect variance (variance of case fatality between all studies).

To estimate the association between age and mortality after a severe exacerbation, the relative risks of age on mortality within a study, if reported, were extracted from the retrieved references. The association with age within each separate study was investigated because there was little difference in the mean age between the different studies. The weighted average relative risk was calculated using the variance in the individual studies as a weight.

\section{RESULTS}

After the first selection, 60 references were obtained in full (fig. 2). An entire review of these remaining publications resulted in the exclusion of another 44 studies for different reasons (fig. 2). The main reasons for exclusion were that the association between hospitalisation for COPD and mortality was not reported (13 studies) and that the study population consisted of a selective subgroup of hospitalised patients (13 studies). Of the latter 13 studies, six studies included patients admitted to ICU or requiring (non)mechanical ventilation only, three included patients treated in the emergency room or pre-hospital setting only, two included hospitalisations for diagnoses other than COPD, while two studies included patients with a first admission or a very mild exacerbation only.

Of the remaining 16 studies, 10 studies met all inclusion criteria except for the $1.5 \mathrm{yrs}$ of follow-up. Hence, a total of six studies were finally included in the meta-analysis to calculate the case fatality rate [15-20]. None of these studies evaluated the effect of an intervention as they were all cohort studies. For one of these six studies, the study of BREKKE et al. [20], we had access to the patient level data. For the other five studies results were based on the data presented in the article. Characteristics of the included studies are shown in table 1.

\section{Case fatality}

Table 2 presents the results of the curve fitting procedure for each of the selected six studies. Details about the parameter values for each study are presented in the supplementary data. The estimated average case-fatality rate for the individual studies varied between $11.4 \%$ and $19.0 \%$. The overall weighted mean value of the case fatality of an exacerbation was $15.6 \%$ (95\% CI 10.9-20.3\%).

\section{Association between mortality and age}

All of the six studies included in the meta-analysis reported on the association between mortality after a hospitalisation for an exacerbation and age. Age was a significant predictor of mortality in univariate analyses (five studies) and remained an independent predictor after correction for other explanatory variables in multivariate analyses (4 studies). On average the probability of dying after hospitalisation for an exacerbation increased by $4.1 \%$ per year increase in age (RR $1.041,95 \%$ CI 1.037-1.045) (six studies).

\section{Average mortality rates at different time-points presented in the literature}

Characteristics of the 10 studies with an insufficient length of follow-up are shown in table $3[9,10,21-28]$. Table 4 shows the average mortality probabilities at different time-points for these 10 studies as well as the six studies that were included in the meta-analysis. Based on all 16 studies combined, the average in-hospital mortality rate was $6.7 \%$. The average mortality rates at 3 and 6 months were $18 \%$ and $26 \%$, respectively.

\section{DISCUSSION}

In this study the case fatality of an exacerbation was calculated by extrapolating the survival curve during the stable period to the time of exacerbation onset. The weighted average casefatality rate was estimated to be $15.6 \%$, with the individual studies varying from $11.4 \%$ to $19.0 \%$. The average in-hospital mortality rate was $6.7 \%$, which strongly supports the notion that the critical period indeed exceeds the duration of the hospitalisation.

\begin{tabular}{|c|c|c|}
\hline First author [ref.] & Patients $\mathrm{n}$ & $\begin{array}{c}\text { Estimated mean case fatality \% } \\
\qquad(95 \% \mathrm{Cl})\end{array}$ \\
\hline Connors [15] & 1016 & $17.2(11.5-23.1)$ \\
\hline Vestbo [16] & 487 & $12.3(5.8-18.4)$ \\
\hline GRoenEWEGEN [17] & 171 & $17.7(10.2-25.8)$ \\
\hline Gunen [18] & 205 & $16.7(7.9-25.4)$ \\
\hline McGHAN [19] & 54269 & $11.4(10.6-12.2)$ \\
\hline BREKKE [20] & 996 & $19.0(18.7-19.3)^{\circ}$ \\
\hline Overall estimate ${ }^{\#}$ & & $15.6(10.9-20.3)$ \\
\hline
\end{tabular}




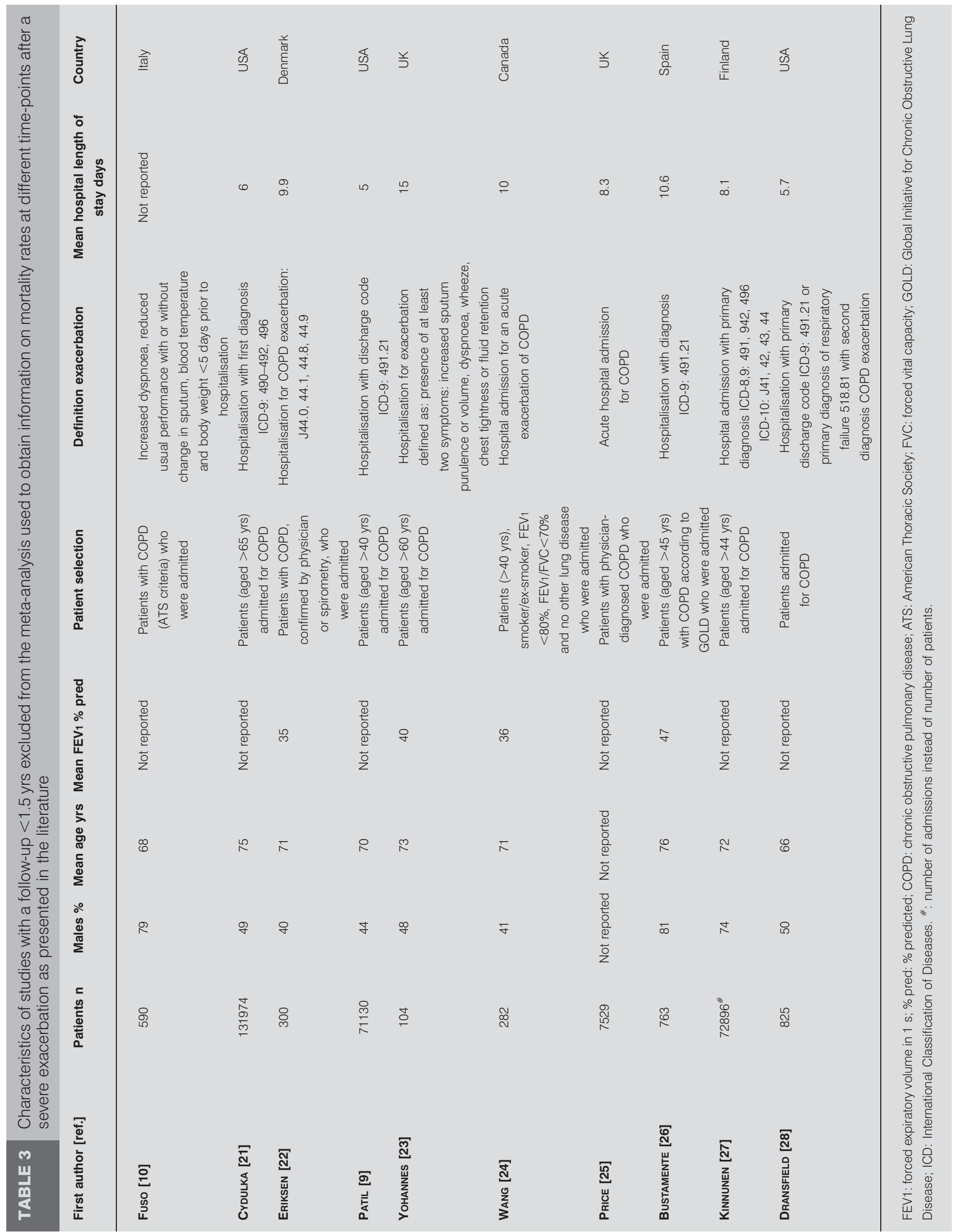




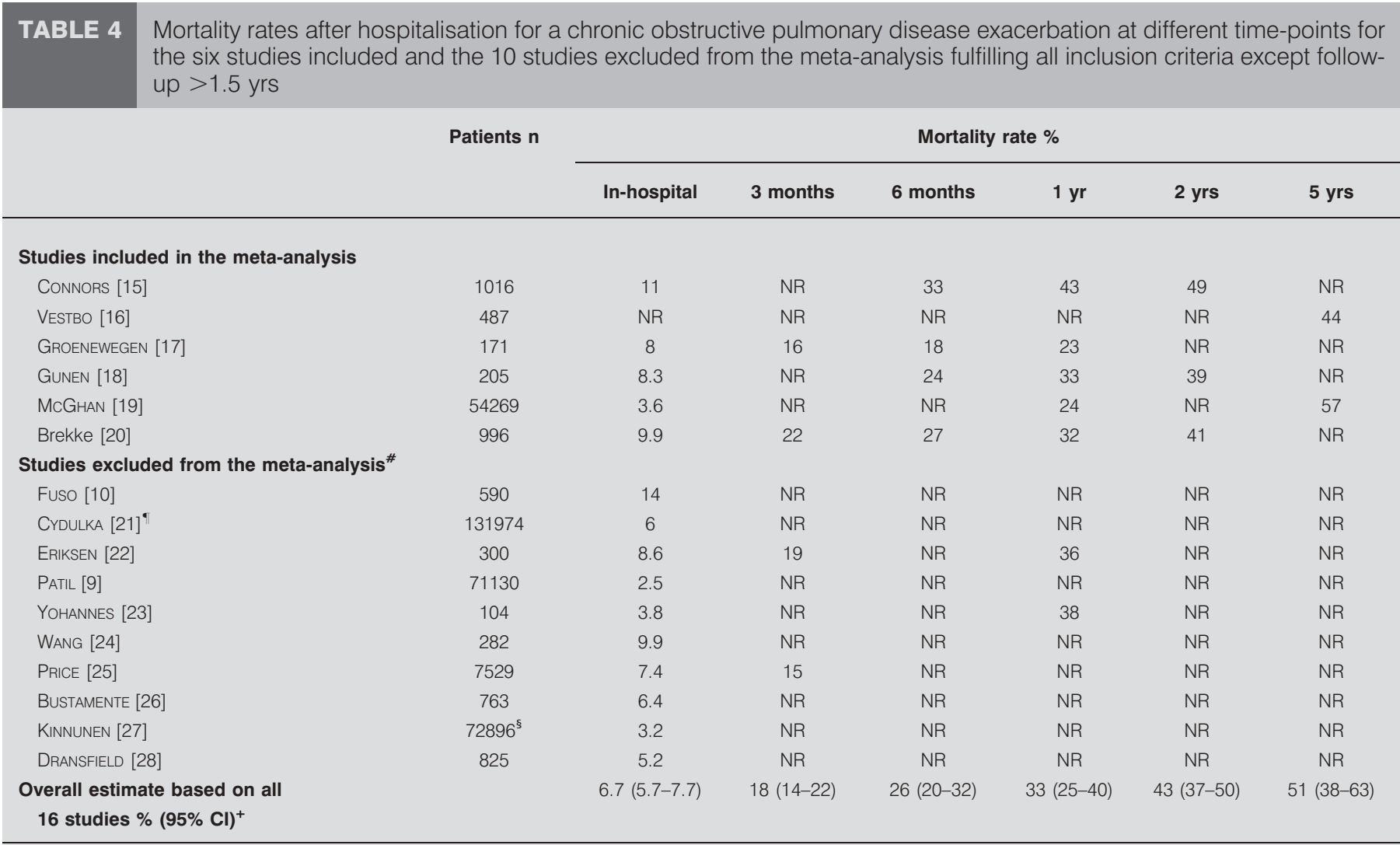

NR: not reported. ${ }^{*}$ : follow-up $<1.5$ yrs; ${ }^{\bullet}$ : results year $1991 ;{ }^{+}$: overall weighted average mortality rates based on random effects analysis; ${ }^{\varsigma}$ : number of admissions instead of number of patients.

However, we would like to emphasise that the estimated case fatality can not be compared with the mortality rates at different time-points as these represent different concepts. The case fatality was calculated as one minus the survival that would have been expected if the patient would have been stable (fig. 1), while mortality at a certain time-points was calculated as one minus the survival at that specific point in time. This also implies that the exact distinction between the critical and stable period after exacerbation onset, however, could not be determined by comparing the case-fatality rate with mortality rates at different points in time. The critical period was defined as the period in which mortality is increased compared to the stable situation. Therefore, this period ranges from the hospital admission until the point were the estimated survival curve during the stable period approaches the actual observed survival curve (fig. 1). Estimating the point where the two survival curves approach each other is only possible if patient-level data are available or when we make additional assumptions on how the case fatality changes over time within the critical period. We had patient-level data from one study, the study of BREKKE et al. [20]. For this study the critical period was estimated to last 4.4 months. The length of the critical period is likely to vary according to the population studied; in patients with several comorbidities the exacerbation may have both more severe $[9,19]$ and longer lasting impact and similarly the critical period could last longer in the elderly.
Due to limited data and the homogeneity of the different studies we were not able to specify the case fatality by subgroups, such as COPD severity (defined by lung function), sex or age. Therefore, we searched for information about the association of these variables with mortality within the extracted studies. Within the studies the relationship of mortality due to an exacerbation with disease severity or sex was less clear. Mortality after hospitalisation for an exacerbation was, however, highly dependent on age (RR 1.041 per increase in year of age).

As the study populations of the six studies selected for the meta-analysis were almost the same with respect to the mean age, 65-71 yrs, age did not influence the between-study comparison of case fatalities. The studies included have sampled data spanning a time-period of $>10$ yrs but no obvious pattern of change over time in case fatality can be seen. This could be the result of the variation in treatment and management between the different countries but was actually also observed in one of the included studies [16]. In contrast, a recent study found indications of a slight improvement of exacerbation-related mortality over time [29].

Despite the homogeneity between the studies with respect to age, the study populations may have differed on other aspects. Although we selected studies from Western countries, the criteria used for hospitalisation, for example, are not similar across countries. This is related to local treatment patterns, which in turn may be driven by local guidelines, medical traditions, 
cultural aspects, financing and reimbursement schemes, etc. In our selected studies the mean length of stay was significantly longer in the European studies compared to studies from the USA, 11 days versus 7 days. However, the mean in-hospital mortality rate did not differ. One study aspect which seemed to have an influence on the results was whether or not patients included in the study had physician- or spirometry-confirmed COPD. Studies including patients with confirmed COPD reported higher mortality rates than studies including patients with hospitalisation for COPD based on International Classification of Disease (ICD) coding. The mean in-hospital mortality rate for both groups were $9.2 \%$ (95\% CI 7.4-10.9\%) and $4.8 \%$ (95\% CI 3.5-6.1\%), respectively. Two of the studies used in the meta-analysis included patients with a hospitalisation for COPD based on ICD coding. If the largest of these two studies, the study of McGHAN [19], was excluded from the meta-analysis, the average case-fatality rate would have been higher, i.e. $17.9 \%(95 \%$ CI $15.8-20 \%$ ). Studies using ICD coding only to define COPD may report lower mortality rates because they also included mild patients or patients with, for example, asthma that were wrongly coded.

In conclusion, mortality in COPD is common and severe exacerbations of COPD are one of the major causes of death in COPD. In this study the case-fatality rate of a severe exacerbation resulting in hospitalisation was estimated to be $15.6 \%$, showing the substantial impact of exacerbations on mortality.

\section{SUPPORT STATEMENT}

This study was financially sponsored by the Netherlands Asthma Foundation (3.4.06.059).

\section{STATEMENT OF INTEREST}

Statements of interest for J. Vestbo and T.L. Feenstra can be found at www.erj.ersjournals.com/site/misc/statements.xhtml

\section{ACKNOWLEDGEMENTS}

We would like to thank P.H. Brekke (Dept of Medicine and Faculty Division, Akershus University Hospital, Lørenskog, Norway) and R. McGhan (Dept of Medicine, Division of Pulmonary Scienced and Critical Care Medicine, Denver Health and Hospital Authority, Denver, CO, USA, ) for the additional information and data they provided. The authors also thank M. Al (Erasmus University, iMTA, Rotterdam, the Netherlands) for her help with the statistical analyses.

\section{REFERENCES}

1 Lopez AD, Shibuya K, Rao C, et al. Chronic obstructive pulmonary disease: current burden and future projections. Eur Respir J 2006; 27: 397-412.

2 Murray CJ, Lopez AD. Alternative projections of mortality and disability by cause 1990-2020: Global Burden of Disease Study. Lancet 1997; 349: 1498-1504.

3 Anthonisen NR, Skeans MA, Wise RA, et al. The effects of a smoking cessation intervention on 14.5-year mortality: a randomized clinical trial. Ann Intern Med 2005; 142: 233-239.

4 Celli BR, Cote CG, Marin JM, et al. The body-mass index, airflow obstruction, dyspnea, and exercise capacity index in chronic obstructive pulmonary disease. N Engl J Med 2004; 350: 1005-1012.

5 Cazzola M, MacNee W, Martinez FJ, et al. Outcomes for COPD pharmacological trials: from lung function to biomarkers. Eur Respir J 2008; 31: 416-469.
6 Zielinski J, MacNee W, Wedzicha J, et al. Causes of death in patients with COPD and chronic respiratory failure. Monaldi Arch Chest Dis 1997; 52: 43-47.

7 Stevens RJ, Coleman RL, Adler AI, et al. Risk factors for myocardial infarction case fatality and stroke case fatality in type 2 diabetes: UKPDS 66. Diabetes Care 2004; 27: 201-207.

8 Faustini A, Marino C, D'Ippoliti D, et al. The impact on risk-factor analysis of different mortality outcomes in COPD patients. Eur Respir J 2008; 32: 629-636.

9 Patil SP, Krishnan JA, Lechtzin N, et al. In-hospital mortality following acute exacerbations of chronic obstructive pulmonary disease. Arch Intern Med 2003; 163: 1180-1186.

10 Fuso L, Incalzi RA, Pistelli R, et al. Predicting mortality of patients hospitalized for acutely exacerbated chronic obstructive pulmonary disease. Am J Med 1995; 98: 272-277.

11 Seneff MG, Wagner DP, Wagner RP, et al. Hospital and 1-year survival of patients admitted to intensive care units with acute exacerbation of chronic obstructive pulmonary disease. JAMA 1995; 274: 1852-1857.

12 Feenstra TL, Van Genugten ML, Hoogenveen RT, et al. The impact of aging and smoking on the future burden of chronic obstructive pulmonary disease: a model analysis in the Netherlands. Am J Respir Crit Care Med 2001; 164: 590-596.

13 Hoogendoorn M, Rutten-van Molken MP, Hoogenveen RT, et al. A dynamic population model of disease progression in COPD. Eur Respir J 2005; 26: 223-233.

14 Der Simonian R, Laird N. Meta-analysis in clinical trials. Control Clin Trials 1986; 7: 177-188.

15 Connors AF, Dawson NV, Thomas C, et al. Outcomes following acute exacerbation of severe chronic obstructive lung disease. The SUPPORT investigators (Study to Understand Prognoses and Preferences for Outcomes and Risks of Treatments). Am J Respir Crit Care Med 1996; 154: 959-967.

16 Vestbo J, Prescott E, Lange $\mathrm{P}$, et al. Vital prognosis after hospitalization for COPD: a study of a random population sample. Respir Med 1998; 92: 772-776.

17 Groenewegen KH, Schols AM, Wouters EF. Mortality and mortality-related factors after hospitalization for acute exacerbation of COPD. Chest 2003; 124: 459-467.

18 Gunen H, Hacievliyagil SS, Kosar F, et al. Factors affecting survival of hospitalised patients with COPD. Eur Respir J 2005; 26: 234-241.

19 McGhan R, Radcliff T, Fish R, et al. Predictors of rehospitalization and death after a severe exacerbation of COPD. Chest 2007; 132: 1748-1755.

20 Brekke PH, Omland T, Holmedal SH, et al. Troponin T elevation and long-term mortality after chronic obstructive pulmonary disease exacerbation. Eur Respir J 2008; 31: 563-570.

21 Cydulka RK, McFadden ER Jr, Emerman CL, et al. Patterns of hospitalization in elderly patients with asthma and chronic obstructive pulmonary disease. Am J Respir Crit Care Med 1997; 156: $1807-1812$

22 Eriksen N, Hansen EF, Munch EP, et al. [Chronic obstructive pulmonary disease. Admission, course and prognosis]. Ugeskr Laeger 2003; 165: 3499-3502.

23 Yohannes AM, Baldwin RC, Connolly MJ. Predictors of 1-year mortality in patients discharged from hospital following acute exacerbation of chronic obstructive pulmonary disease. Age Ageing 2005; 34: 491-496.

24 Wang Q, Bourbeau J. Outcomes and health-related quality of life following hospitalization for an acute exacerbation of COPD. Respirology 2005; 10: 334-340.

25 Price LC, Lowe D, Hosker HS, et al. UK National COPD Audit 2003: Impact of hospital resources and organisation of care on patient outcome following admission for acute COPD exacerbation. Thorax 2006; 61: 837-842.

26 Bustamante-Fermosel A, De Miguel-Yanes JM, Duffort-Falco M, et al. Mortality-related factors after hospitalization for acute 
exacerbation of chronic obstructive pulmonary disease: the burden of clinical features. Am J Emerg Med 2007; 25: 515-522.

27 Kinnunen T, Saynajakangas O, Keistinen T. Features of hospitalisations for acute exacerbation of COPD resulting in death Monaldi Arch Chest Dis 2007; 67: 10-14.
28 Dransfield MT, Rowe SM, Johnson JE, et al. Use of beta blockers and the risk of death in hospitalised patients with acute exacerbations of COPD. Thorax 2008; 63: 301-305.

29 Eriksen N, Vestbo J. Management and survival of patients admitted with an exacerbation of COPD: Comparison of two Danish patient cohorts. Clin Respir J 2010; 4: 208-214. 